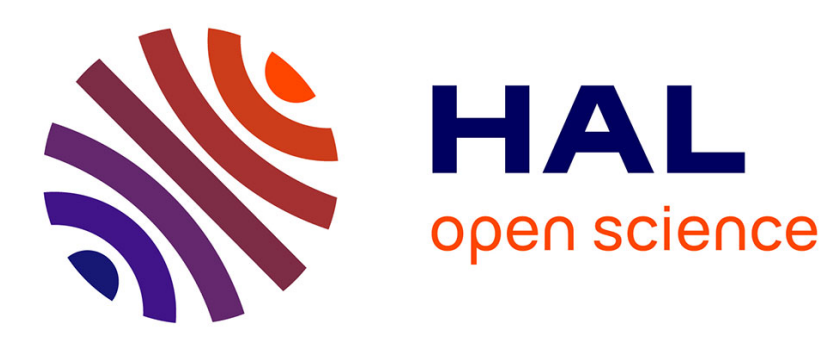

\title{
Spectroscopie micro-onde de 32SF5 35Cl
}

Jocelyn Chanussot, R. Jurek, J. Bellet

\section{To cite this version:}

Jocelyn Chanussot, R. Jurek, J. Bellet. Spectroscopie micro-onde de 32SF5 35Cl. Journal de Physique, 1976, 37 (10), pp.1129-1134. 10.1051/jphys:0197600370100112900 . jpa-00208511

\section{HAL Id: jpa-00208511 https://hal.science/jpa-00208511}

Submitted on 1 Jan 1976

HAL is a multi-disciplinary open access archive for the deposit and dissemination of scientific research documents, whether they are published or not. The documents may come from teaching and research institutions in France or abroad, or from public or private research centers.
L'archive ouverte pluridisciplinaire HAL, est destinée au dépôt et à la diffusion de documents scientifiques de niveau recherche, publiés ou non, émanant des établissements d'enseignement et de recherche français ou étrangers, des laboratoires publics ou privés. 
Classification

Physics Abstracts

5.445

\title{
SPECTROSCOPIE MICRO-ONDE DE ${ }^{32} \mathrm{SF}_{5}{ }^{35} \mathrm{Cl}$
}

\author{
J. CHANUSSOT, R. JUREK \\ Laboratoire de Spectroscopie Hertzienne (*), Faculté des Sciences MIPC \\ 6, bd Gabriel, 21000 Dijon, France \\ et J. BELLET \\ Laboratoire de Spectroscopie Hertzienne $(* *)$, Université de Lille, I, \\ B.P. 36, 59650 Villeneuve-d'Ascq, France
}

(Reçu le 2 mars 1976, accepté le 17 mai 1976)

\begin{abstract}
Résumé. - L'étude des spectres d'absorption de la molécule ${ }^{32} \mathrm{SF}_{5}{ }^{35} \mathrm{Cl}$ à grande résolution et hautes fréquences (largeur Doppler de $170 \mathrm{kHz}$ à $210 \mathrm{GHz}$ ) permet la détermination précise de ses paramètres moléculaires. Les fréquences calculées à partir de ceux-ci conduisent à des écarts types de l’ordre de $35 \mathrm{kHz}$. Les résultats antérieurs sont discutés.
\end{abstract}

Abstract. - The high resolution study of the rotational spectra of ${ }^{32} \mathrm{SF}_{5}{ }^{35} \mathrm{Cl}$ in the high frequency ranges (Doppler width about $170 \mathrm{kHz}$ at $210 \mathrm{GHz}$ ) gives a good set of molecular parameters. Calculated frequencies give standard deviations of the order of $35 \mathrm{kHz}$. Previous results are discussed.

1. Introduction. - Dans le cadre de l'étude spectroscopique des molécules à symétrie $\mathrm{C}_{4 \mathrm{v}}$, un travail antérieur avait permis de déterminer un ensemble de paramètres moléculaires pour $\mathrm{SF}_{5} \mathrm{Cl}$ [1]. En particulier, l'interprétation des spectres observés à $70 \mathrm{GHz}$ faisait intervenir les termes de distorsion centrifuge du $6^{e}$ ordre provenant de $\mathrm{h}_{4}^{+}$[2], [6]. Dans le but de préciser la validité de ces paramètres, des spectres ont été relevés à des fréquences élevées $(210 \mathrm{GHz})$; leur interprétation fait l'objet du présent travail.

2. Considérations générales. - L'étude d'une molécule toupie symétrique lourde $\left({ }^{32} \mathrm{SF}_{5}{ }^{35} \mathrm{Cl}\right.$ possède une masse de 162) et de faible moment dipolaire $(\mu=0,51 \mathrm{D})$ présente de nombreuses difficultés. D'une manière générale les spectres observés sont mal résolus et peu intenses. D'autre part, encore peu de résultats expérimentaux sont connus pour les molécules à symétrie $C_{4 v}$. D'après le formalisme habituel, les fréquences des raies d'absorption rotationnelle relatives aux branches $\mathbf{R}$ parallèles sont données par :

$$
\begin{aligned}
v_{I, J, K, F} & =2 B_{0}(J+1)-4 D_{J}(J+1)^{3}+ \\
& +e q Q\left(f_{I, J, F}-f_{I, J+1, F+1}\right)-2 D_{J K}(J+1) K^{2} \\
& +\frac{3 e q Q}{J+1}\left[\frac{f_{I, J+1, F+1}}{J+2}-\frac{f_{I, J, F}}{J}\right] K^{2} .
\end{aligned}
$$

(*) Equipe de recherche associée au C.N.R.S.

(**) Laboratoire associé au C.N.R.S.
On constate que les structures caractéristiques des spectres sont dues à des contributions compétitives

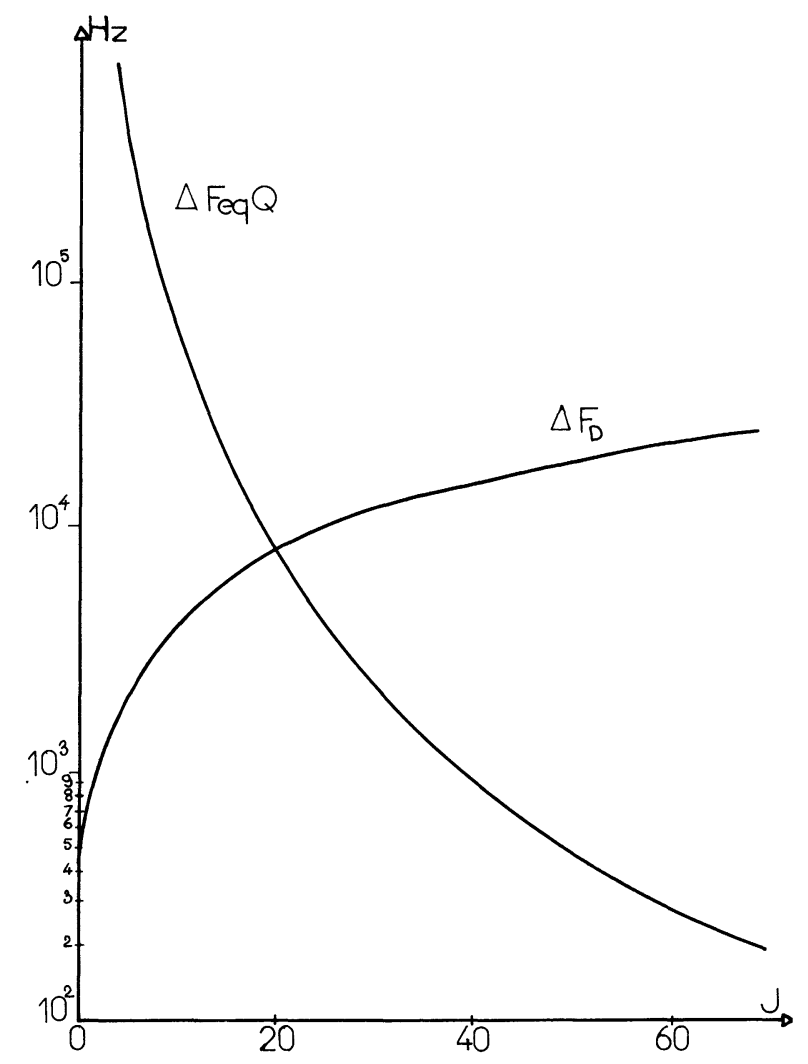

FIG. 1. - Contributions respectives de la distorsion centrifuge et du couplage quadripolaire. 
$\Delta F_{D}$ du paramètre de distorsion centrifuge $D_{J K}$ et $\Delta F_{e q Q}$ du paramètre de couplage quadripolaire $e q Q$ :

$$
\begin{aligned}
\Delta F_{D} & =\left|-2 D_{J K}(J+1)\right| \\
\Delta F_{e q Q} & =\left|\frac{3 e q Q}{J+1}\left(\frac{f_{I, J+1, F+1}}{J+2}-\frac{f_{I, J, F}}{J}\right)\right| .
\end{aligned}
$$

Ces contributions respectives varient en fonction de la fréquence de travail, donc de $J$ suivant le schéma de la figure 1. Ceci nous conduit aux constatations suivantes :

1) A $8 \mathrm{~mm}$ de longueur d'onde $(J \simeq 10)$ la contribution de $e q Q$ est prépondérante et les spectres théoriques sont bien résolus mais l'intensité est très faible.

2) Les spectres théoriques à $4 \mathrm{~mm}(J \simeq 20)$ doivent être particulièrement complexes et mal résolus du fait des contributions équivalentes de $D_{J K}$ et $e q Q$.

3) Ceci nous conduit tout naturellement à reprendre les travaux antérieurs à des fréquences supérieures $(J \simeq 60)$ où la contribution de la distorsion centrifuge est prépondérante. Dans cette zone la résolution n'est plus limitée que par l'effet Doppler; de plus, la valeur optimum de $J$ du point de vue intensité des spectres [3] se situe au voisinage de $J=58$.

3. Résultats expérimentaux. - Le spectromètre utilisé est du type vidéo. La cellule d'absorption est un élément de $6 \mathrm{~m}$ de longueur (guide $\mathrm{X}, 3 \mathrm{~cm}$ ) maintenu à $-80^{\circ} \mathrm{C}$. La fréquence de travail est obtenue, à partir d'un klystron source, par multiplication par diode à pointe. La pression du gaz est ajustée de façon telle que la résolution limite Doppler soit atteinte; ici, dans la gamme des $200 \mathrm{GHz}$, la largeur Doppler est $\Delta v_{\mathrm{D}} \simeq 160 \mathrm{kHz}$.

Nous donnons dans le tableau I les transitions mesurées et nous indiquons pour chaque transition $J \rightarrow J+1$ les fréquences correspondant aux valeurs extrêmes de $|K|$ observé.

L'analyse détaillée de la transition $J=58 \rightarrow 59$ donnée à titre d'exemple (tableau II) suggère un certain nombre de remarques :

TABLEAU I

Transitions rotationnelles de ${ }^{32} \mathrm{SF}_{5}{ }^{35} \mathrm{Cl}$ mesurées vers $1,4 \mathrm{~mm}$ de longueur d'onde

Transitions

$J \rightarrow J+1$

$J=55 \rightarrow 56$

$\Delta=2,1 \mathrm{kHz}$

$\sigma=45 \mathrm{kHz}$

$J=56 \rightarrow 57$

$\Delta=1,7 \mathrm{kHz}$

$\sigma=39 \mathrm{kHz}$

$J=57 \rightarrow 58$

$\Delta=2,2 \mathrm{kHz}$

$\sigma=38 \mathrm{kHz}$

$J=58 \rightarrow 59$

$\Delta=6 \mathrm{kHz}$

$\sigma=28 \mathrm{kHz}$

$J=59 \rightarrow 60$

$\Delta=-14 \mathrm{kHz}$

$\sigma=26 \mathrm{kHz}$

$J=60 \rightarrow 61$

$\Delta=2,3 \mathrm{kHz}$

$\sigma=28 \mathrm{kHz}$

$J=65 \rightarrow 66$

$\Delta=1,5 \mathrm{kHz}$

$\sigma=38 \mathrm{kHz}$
Raie obs. à la fréq. la plus haute $(\mathrm{MHz})$

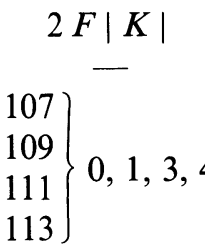

Raie obs. à la fréq. la plus basse $(\mathrm{MHz})$

$2 F|K|$

204275,526

211565,212

109

111

113

0,1

207855,171

115

111

$\left.\begin{array}{l}113 \\ 115\end{array}\right\}$

115

$0,1,3,4$

211496,275

113

115

117

0,1

215139,817

119 )

115

117

119

121

$0,1,3$

218839,029

117

119

121

123

127

129

131
133

$0,1,3$

$0,1,3$

222421,083

240700,725 $\left.\begin{array}{l}111 \\ 113\end{array}\right\} 55$

$\left.\begin{array}{l}113 \\ 115\end{array}\right\} 56$

$\left.\begin{array}{l}115 \\ 117\end{array}\right\} 56$

$\left.\begin{array}{l}117 \\ 119\end{array}\right\} 26$

$\left.\begin{array}{l}119 \\ 121\end{array}\right\} 58$

127

129

131

109

$111\} 55$

56

133

( $\Delta$ écart moyen ; $\sigma$ écart type) 
TABLEAU II

Transition $J=58 \rightarrow 59 \mathrm{de}^{32} \mathrm{SF}_{5}{ }^{35} \mathrm{Cl}$

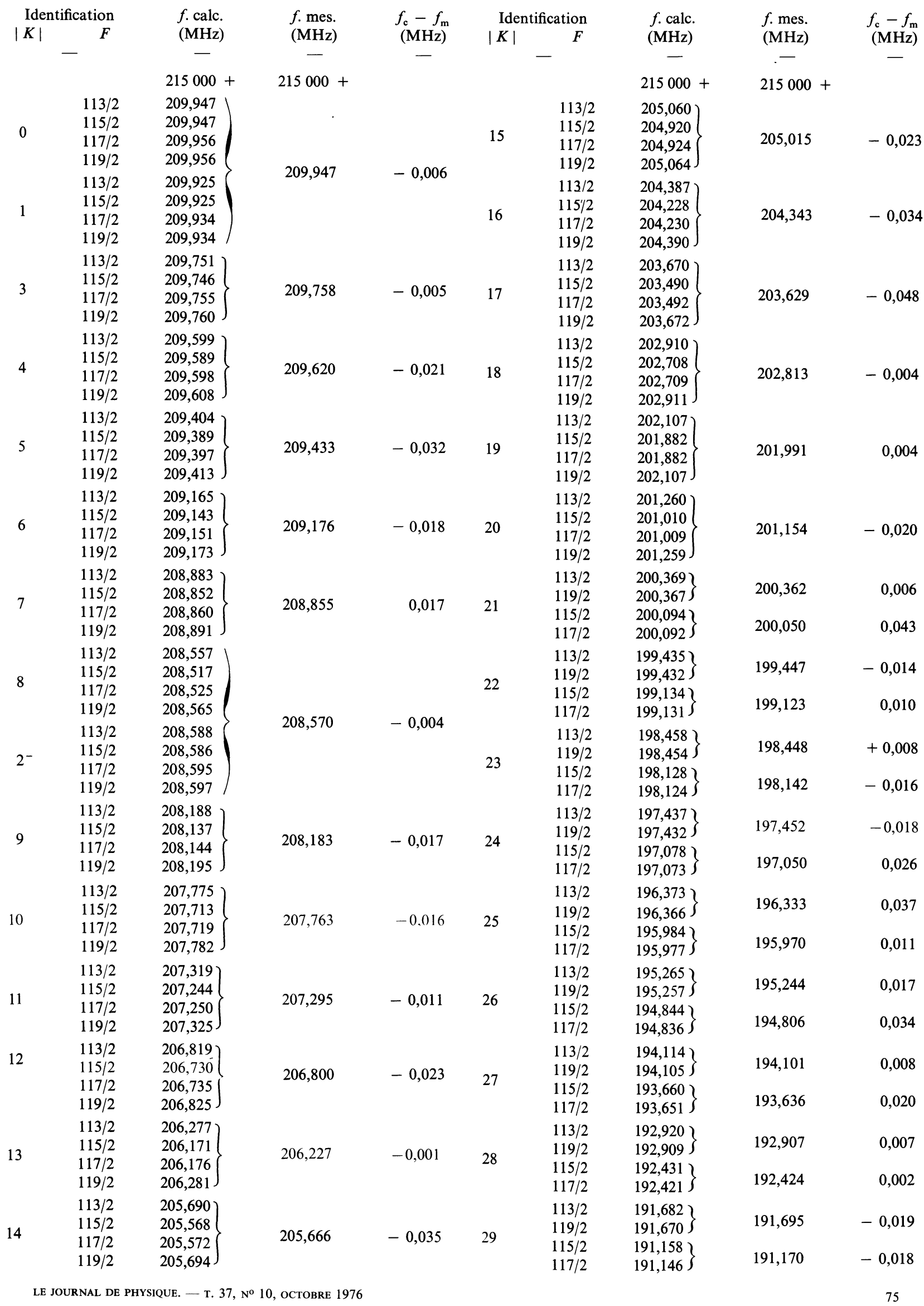


TABLEAU II

\begin{tabular}{|c|c|c|c|c|c|c|c|c|c|}
\hline \multicolumn{2}{|c|}{ Identification } & \multirow{2}{*}{$\begin{array}{l}f . \text { calc. } \\
(\mathrm{MHz})\end{array}$} & \multirow{2}{*}{$\begin{array}{l}f \text {. mes. } \\
(\mathrm{MHz})\end{array}$} & \multirow{2}{*}{$\begin{array}{c}f_{\mathrm{c}}-f_{\mathrm{m}} \\
(\mathrm{MHz})\end{array}$} & \multicolumn{2}{|c|}{ Identification } & \multirow{2}{*}{$\begin{array}{l}f . \text { calc. } \\
(\mathrm{MHz})\end{array}$} & \multirow{2}{*}{$\begin{array}{l}f \text {. mes. } \\
\text { (MHz) }\end{array}$} & \multirow{2}{*}{$\begin{array}{c}f_{\mathrm{c}}-f_{\mathrm{m}} \\
(\mathrm{MHz})\end{array}$} \\
\hline$|K|$ & $F$ & & & & $|K|$ & $F$ & & & \\
\hline \multirow[b]{2}{*}{30} & $\begin{array}{l}113 / 2 \\
119 / 2\end{array}$ & $\left.\begin{array}{l}190,401 \\
190,387\end{array}\right\}$ & 190,429 & $-0,035$ & \multirow[b]{2}{*}{44} & $\begin{array}{l}113 / 2 \\
119 / 2\end{array}$ & $\left.\begin{array}{l}167,901 \\
167,860\end{array}\right\}$ & 167,857 & 0,023 \\
\hline & $\begin{array}{l}115 / 2 \\
117 / 2\end{array}$ & $\left.\begin{array}{l}189,840 \\
189,826\end{array}\right\}$ & 189,835 & $-0,002$ & & $\begin{array}{l}115 / 2 \\
117 / 2\end{array}$ & $\left.\begin{array}{l}166,694 \\
166,654\end{array}\right\}$ & 166,657 & 0,017 \\
\hline \multirow[b]{2}{*}{31} & $\begin{array}{l}113 / 2 \\
119 / 2\end{array}$ & $\left.\begin{array}{l}189,076 \\
189,060\end{array}\right\}$ & 189,049 & 0,019 & \multirow[b]{2}{*}{45} & $\begin{array}{l}113 / 2 \\
119 / 2\end{array}$ & $\left.\begin{array}{l}165,968 \\
165,925\end{array}\right\}$ & 165,892 & 0,054 \\
\hline & $\begin{array}{l}115 / 2 \\
117 / 2\end{array}$ & $\left.\begin{array}{l}188,477 \\
188,462\end{array}\right\}$ & 188,449 & 0,021 & & $\begin{array}{l}115 / 2 \\
117 / 2\end{array}$ & $\left.\begin{array}{l}164,706 \\
164,663\end{array}\right\}$ & 164,683 & 0,001 \\
\hline \multirow{2}{*}{32} & $\begin{array}{l}113 / 2 \\
119 / 2\end{array}$ & $\left.\begin{array}{l}187,708 \\
187,691\end{array}\right\}$ & 187,720 & $-0,021$ & \multirow{2}{*}{46} & $\begin{array}{l}113 / 2 \\
119 / 2\end{array}$ & $\left.\begin{array}{l}163,991 \\
163,946\end{array}\right\}$ & 163,975 & $-0,007$ \\
\hline & $\begin{array}{l}115 / 2 \\
117 / 2\end{array}$ & $\left.\begin{array}{l}187,070 \\
187,053\end{array}\right\}$ & 187,036 & 0,026 & & $\begin{array}{l}115 / 2 \\
117 / 2\end{array}$ & $\left.\begin{array}{l}162,673 \\
162,628\end{array}\right\}$ & 162,652 & $-0,002$ \\
\hline \multirow[b]{2}{*}{33} & $\begin{array}{l}113 / 2 \\
119 / 2\end{array}$ & $\left.\begin{array}{l}186,296 \\
186,277\end{array}\right\}$ & 186,259 & 0,028 & \multirow{2}{*}{47} & $\begin{array}{l}113 / 2 \\
119 / 2\end{array}$ & $\left.\begin{array}{l}161,972 \\
161,924\end{array}\right\}$ & 161,833 & 0,115 \\
\hline & $\begin{array}{l}115 / 2 \\
117 / 2\end{array}$ & $\left.\begin{array}{l}185,617 \\
185,599\end{array}\right\}$ & 185,623 & $-0,015$ & & $\begin{array}{l}115 / 2 \\
117 / 2\end{array}$ & $\left.\begin{array}{l}160,595 \\
160,548\end{array}\right\}$ & 160,579 & $-0,008$ \\
\hline \multirow{2}{*}{34} & $\begin{array}{l}113 / 2 \\
119 / 2\end{array}$ & $\left.\begin{array}{l}184,841 \\
184,820\end{array}\right\}$ & 184,822 & 0,009 & \multirow{2}{*}{48} & $\begin{array}{l}113 / 2 \\
119 / 2\end{array}$ & $\left.\begin{array}{l}159,908 \\
159,859\end{array}\right\}$ & 159,856 & 0,028 \\
\hline & $\begin{array}{l}115 / 2 \\
117 / 2\end{array}$ & $\left.\begin{array}{l}184,121 \\
184,100\end{array}\right\}$ & 184,096 & 0,015 & & $\begin{array}{l}115 / 2 \\
117 / 2\end{array}$ & $\left.\begin{array}{l}158,473 \\
158,423\end{array}\right\}$ & 158,461 & $-0,013$ \\
\hline \multirow[b]{2}{*}{35} & $\begin{array}{l}113 / 2 \\
119 / 2\end{array}$ & $\left.\begin{array}{l}183,342 \\
183,320\end{array}\right\}$ & 183,295 & 0,036 & \multirow[b]{2}{*}{49} & $\begin{array}{l}113 / 2 \\
119 / 2\end{array}$ & $\left.\begin{array}{l}157,802 \\
157,750\end{array}\right\}$ & 157,726 & 0,050 \\
\hline & $\begin{array}{l}115 / 2 \\
117 / 2\end{array}$ & $\left.\begin{array}{l}182,579 \\
182,557\end{array}\right\}$ & 182,599 & $-0,031$ & & $\begin{array}{l}115 / 2 \\
117 / 2\end{array}$ & $\left.\begin{array}{l}156,306 \\
156,254\end{array}\right\}$ & 156,358 & $-0,078$ \\
\hline \multirow{2}{*}{36} & $\begin{array}{l}113 / 2 \\
119 / 2\end{array}$ & $\left.\begin{array}{l}181,800 \\
181,776\end{array}\right\}$ & 181,816 & $-0,028$ & \multirow[b]{2}{*}{50} & $\begin{array}{l}113 / 2 \\
119 / 2\end{array}$ & $\left.\begin{array}{l}155,652 \\
155,597\end{array}\right\}$ & 155,593 & 0,032 \\
\hline & $\begin{array}{l}115 / 2 \\
117 / 2\end{array}$ & $\left.\begin{array}{l}180,993 \\
180,969\end{array}\right\}$ & 180,979 & 0,002 & & $\begin{array}{l}115 / 2 \\
117 / 2\end{array}$ & $\left.\begin{array}{l}154,094 \\
154,039\end{array}\right\}$ & 154,093 & $-0,027$ \\
\hline \multirow{2}{*}{37} & $\begin{array}{l}113 / 2 \\
119 / 2\end{array}$ & $\left.\begin{array}{l}180,215 \\
180,189\end{array}\right\}$ & 180,187 & 0,015 & \multirow{2}{*}{51} & $\begin{array}{l}113 / 2 \\
119 / 2\end{array}$ & $\left.\begin{array}{l}153,458 \\
153,401\end{array}\right\}$ & 153,427 & 0,003 \\
\hline & $\begin{array}{l}115 / 2 \\
117 / 2\end{array}$ & $\left.\begin{array}{l}179,362 \\
179,336\end{array}\right\}$ & 179,311 & 0,038 & & $\begin{array}{l}115 / 2 \\
117 / 2\end{array}$ & $\left.\begin{array}{l}151,838 \\
151,780\end{array}\right\}$ & 151,792 & 0,017 \\
\hline \multirow{2}{*}{38} & $\begin{array}{l}113 / 2 \\
119 / 2\end{array}$ & $\left.\begin{array}{l}178,586 \\
178,558\end{array}\right\}$ & 178,588 & $-0,016$ & \multirow[b]{2}{*}{52} & $\begin{array}{l}113 / 2 \\
119 / 2\end{array}$ & $\left.\begin{array}{l}151,221 \\
151,161\end{array}\right\}$ & 151,144 & 0,047 \\
\hline & $\begin{array}{l}115 / 2 \\
117 / 2\end{array}$ & $\left.\begin{array}{l}177,686 \\
177,659\end{array}\right\}$ & 177,661 & 0,012 & & $\begin{array}{l}115 / 2 \\
117 / 2\end{array}$ & $\left.\begin{array}{l}149,536 \\
149,476\end{array}\right\}$ & 149,470 & 0,036 \\
\hline \multirow{2}{*}{39} & $\begin{array}{l}113 / 2 \\
119 / 2\end{array}$ & $\left.\begin{array}{l}176,914 \\
176,884\end{array}\right\}$ & 176,911 & $-0,012$ & \multirow{2}{*}{53} & $\begin{array}{l}113 / 2 \\
119 / 2\end{array}$ & $\left.\begin{array}{l}148,941 \\
148,878\end{array}\right\}$ & 148,852 & 0,058 \\
\hline & $\begin{array}{l}115 / 2 \\
117 / 2\end{array}$ & $\left.\begin{array}{l}175,966 \\
175,936\end{array}\right\}$ & 175,939 & 0,012 & & $\begin{array}{l}115 / 2 \\
117 / 2\end{array}$ & $\left.\begin{array}{l}147,191 \\
147,128\end{array}\right\}$ & 147,124 & 0,036 \\
\hline \multirow{2}{*}{40} & $\begin{array}{l}113 / 2 \\
119 / 2\end{array}$ & $\left.\begin{array}{l}175,198 \\
175,166\end{array}\right\}$ & 175,150 & 0,032 & 54 & $\begin{array}{l}113 / 2 \\
119 / 2\end{array}$ & $\left.\begin{array}{l}146,617 \\
146,552\end{array}\right\}$ & 146,578 & 0,006 \\
\hline & $\begin{array}{l}115 / 2 \\
117 / 2\end{array}$ & $\left.\begin{array}{l}174,201 \\
174,169\end{array}\right\}$ & 174,187 & $-0,002$ & 34 & $\begin{array}{l}115 / 2 \\
117 / 2\end{array}$ & $\left.\begin{array}{l}144,800 \\
144,735\end{array}\right\}$ & 144,745 & 0,022 \\
\hline 41 & $\begin{array}{l}113 / 2 \\
119 / 2\end{array}$ & $\left.\begin{array}{l}173,439 \\
173,405\end{array}\right\}$ & 173,374 & 0,048 & & $\begin{array}{l}113 / 2 \\
119 / 2\end{array}$ & $\left.\begin{array}{l}144,249 \\
144,186\end{array}\right\}$ & 144,196 & 0,022 \\
\hline 71 & $\begin{array}{l}115 / 2 \\
117 / 2\end{array}$ & $\left.\begin{array}{l}172,391 \\
172,358\end{array}\right\}$ & 172,324 & 0,051 & JJ & $\begin{array}{l}115 / 2 \\
117 / 2\end{array}$ & $\left.\begin{array}{l}142,363 \\
142,300\end{array}\right\}$ & 142,321 & 0,011 \\
\hline & $\begin{array}{l}113 / 2 \\
119 / 2\end{array}$ & $\left.\begin{array}{l}171,636 \\
171,600\end{array}\right\}$ & 171,625 & $-0,007$ & & $\begin{array}{l}113 / 2 \\
119 / 2\end{array}$ & $\left.\begin{array}{l}141,838 \\
141,773\end{array}\right\}$ & 141,811 & $-0,006$ \\
\hline 42 & $\begin{array}{l}115 / 2 \\
117 / 2\end{array}$ & $\left.\begin{array}{c}170,537 \\
170,501\end{array}\right\}$ & 170,509 & 0,010 & 56 & $115 / 2$ & $139,883\}$ & & \\
\hline & $\begin{array}{l}117 / 2 \\
113 / 2\end{array}$ & & & s. & & $117 / 2$ & 139,8175 & 139,891 & $-0,041$ \\
\hline & $\begin{array}{l}113 / 2 \\
119 / 2\end{array}$ & $\left.\begin{array}{l}169, / 90 \\
169,752\end{array}\right\}$ & 169,723 & 0,048 & & & & & \\
\hline 43 & $\begin{array}{l}115 / 2 \\
117 / 2\end{array}$ & $\left.\begin{array}{l}168,638 \\
168,600\end{array}\right\}$ & 168,577 & 0,042 & & & $\mathrm{y}$ & $\mathrm{ECa}$ & \\
\hline
\end{tabular}


1) Pour $|K|$ faible, $(|K|<10$, Fig. 2) les quatre composantes quadripolaires (structure en $F$ due à l'atome de chlore) donnent une seule raie pour chaque valeur de $|K|$. Le dédoublement $|K|=2$ est observé [4].

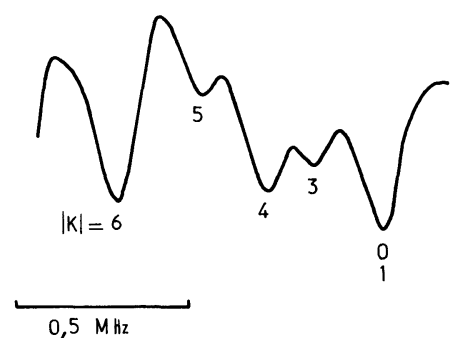

FIG. 2. - Transition $J=58 \rightarrow 59|K|<10$.

2) Pour $10<|K|<18$ (Fig. 3) on observe, pour la première fois à notre connaissance la structure caractéristique liée à la symétrie $\mathrm{C}_{4 \mathrm{v}}$ : les intensités relatives suivent la loi des poids statistiques de spin nucléaire [5]

$$
\begin{array}{ll}
|K|=4 p & I=4 \\
|K|=4 p+2 & I=6 \\
|K|=2 p+1 & I=3 .
\end{array}
$$

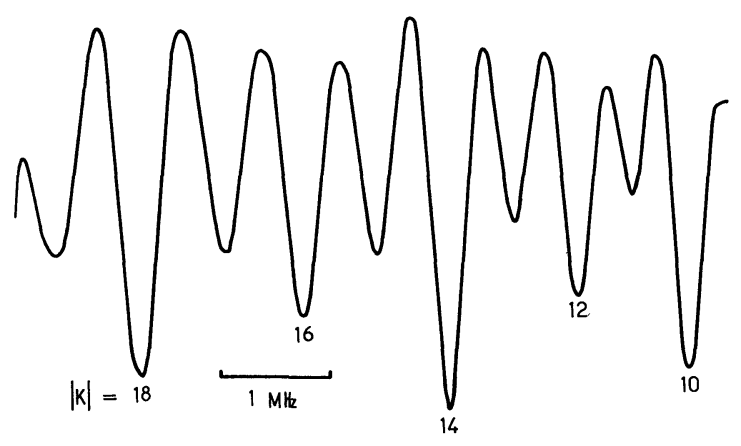

Fig. 3. - Transition $J=58 \rightarrow 5910 \leqslant|K| \leqslant 18$.

3) Pour les valeurs suivantes de $|K|$,

$$
(18<|K|<22 \text {, Fig. 4) }
$$

l'importance croissante de la contribution quadripolaire $e q Q$ fait apparaître progressivement un dédoublement de chaque raie; chaque composante du doublet correspond à deux valeurs de $F: F=113 / 2$

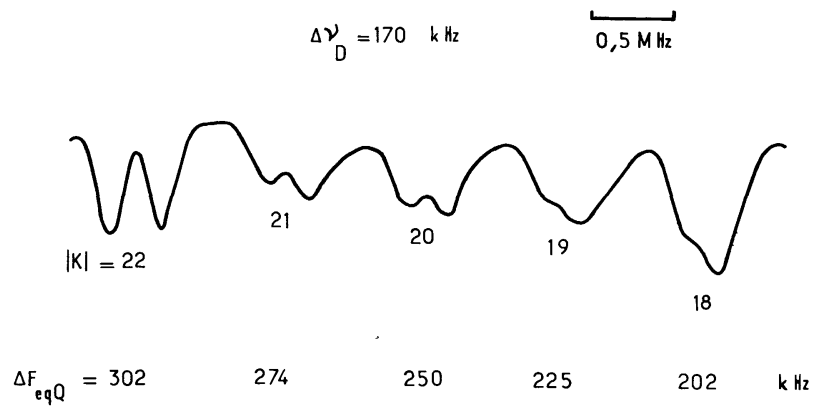

FIG. 4. - Transition $J=58 \rightarrow 5918 \leqslant|K| \leqslant 22$. et $F=119 / 2$ pour la composante à fréquence supérieure ; $F=115 / 2$ et $F=117 / 2$ pour la composante à fréquence inférieure. Pour $|K|=18$ on retrouve effectivement par le calcul que la contribution quadripolaire est sensiblement égale à la largeur Doppler des raies.

4) Pour la zone suivante du spectre (Fig. 5), la structure en doublets est bien résolue; chaque composante du doublet suit la loi caractéristique des intensités relatives. Le rôle du paramètre $D_{J K}$ devient de plus en plus important ce qui explique la plus grande dispersion du spectre.

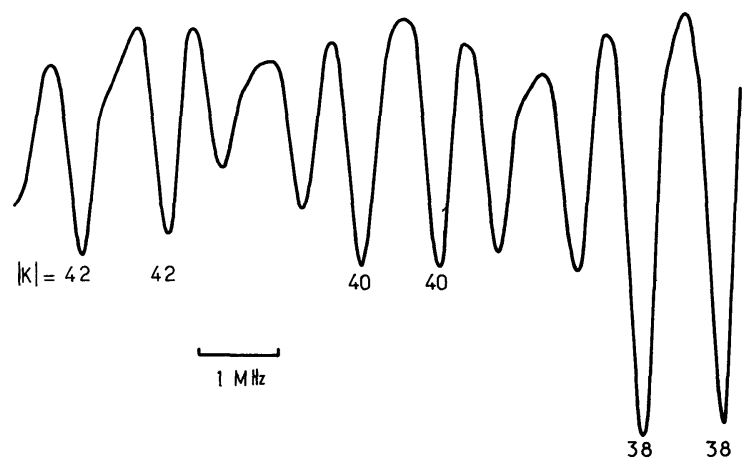

Fig. 5. - Transition $J=58 \rightarrow 5938 \leqslant|K| \leqslant 42$.

3. Calcul des paramètres. Conclusion. - On détermine l'ensemble des paramètres moléculaires par la méthode des moindres carrés portant sur l'ensemble des transitions mesurées, y compris celles déjà publiées par ailleurs [1]. Conformément au tableau I, 400 raies nouvelles observées sont utilisées au total. Dans le cas des structures partiellement résolues, la valeur expérimentale est utilisée autant de fois (sans pondération particulière) qu'il lui est attribuée d'identifications possibles (par exemple 4 fois pour la mesure à $215202,813 \mathrm{MHz}$ attribuée aux raies : $|K|=18$; $F=113 / 2,115 / 2,117 / 2$ et $119 / 2$ ).

Les résultats suivants sont obtenus :

$$
\begin{aligned}
B_{0} & =1824,5924 \pm 0,0001 \mathrm{MHz} \\
D_{J} & =111,94 \pm 0,25 \mathrm{~Hz} \\
D_{J K} & =186,8 \pm 0,2 \mathrm{~Hz} \\
e q Q & =-85,35 \pm 0,2 \mathrm{MHz} \\
R_{6} & =1,55 \pm 0,01 \mathrm{~Hz}
\end{aligned}
$$

Les erreurs indiquées dans ce tableau sont légèrement supérieures aux écarts types afin que l'ensemble des incertitudes associées à chaque paramètre corresponde à l'incertitude expérimentale.

Nous rappelons que le paramètre $R_{6}$ responsable du dédoublement de type $K$ [4] spécifique aux molécules de symétrie $C_{4 \mathrm{v}}$, provient des termes non diagonaux $\langle K, K \pm 4\rangle$, de l'hamiltonien de rotation, qui couplent les niveaux de même énergie $K=2$ et $K=-2$. 
Cette détermination permet de recalculer toutes les transitions conformément à l'exemple du tableau II $\left({ }^{1}\right)$. Les différences $\left|f_{\text {mes }}-f_{\text {cal }}\right|$ restent compatibles avec l'incertitude expérimentale. Aux fréquences élevées $(J \simeq 60)$, contrairement aux conclusions tirées de l'étude des spectres à fréquences plus basses $(J \simeq 20)$ les transitions $v_{I, J, K, F}$ varient linéairement en fonction de $K^{2}$ (Fig. 6); la contribution des termes de distorsion centrifuge d'ordre supérieur (termes $H_{J}$, $H_{J J K}$ et $\left.H_{J K K}\right)$ ne peut donc être mise en évidence pour cette molécule. En conséquence la détermination de ces paramètres est impossible sur la base de ces travaux; leur introduction dans les calculs doit donc être abandonnée. En [1] une valeur pour les paramètres $H$ avait été proposée. Ces résultats auraient dû entraîner une contribution très importante aux fréquences envisagées ici $(210 \mathrm{GHz})$; le fait que $v_{I J K F}$ reste linéaire vis-à-vis de $K^{2}$ nous oblige donc à abandonner les termes d'ordre supérieur. La raison de cette amélioration est due à la meilleure résolution des spectres mesurés à $210 \mathrm{GHz}$.

L'étude est poursuivie à des fréquences supérieures tant pour les états fondamentaux des différentes. variétés isotopiques que pour les divers états excités. h

( ${ }^{1}$ La liste de toutes les fréquences expérimentales et calculées peut être communiquée à toute personne intéressée. Nous ne précisons ici (Tableau I) que les écarts moyens et écarts types obtenus pour chaque transition $J \rightarrow \mathrm{J}+1$.

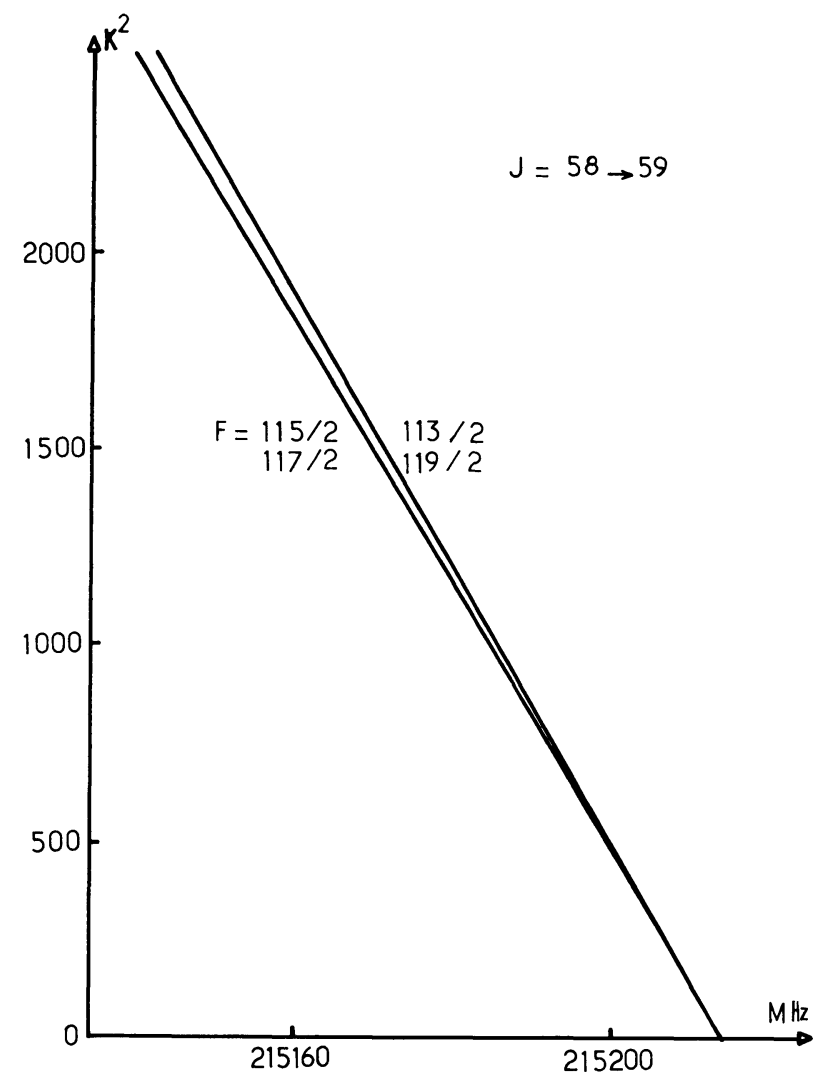

Fig. 6. - Variation de la fréquence en fonction de $K^{2}$.

\section{Bibliographie}

[1] Chanussot, J., J. Phys. 31 (1970) 313.

[2] Tarrago, G., Thèse de Doctorat d'état Paris (1965).

[3] GoRDY, W., CoOK, R. L., Microwave Molecular Spectra (John Wiley and Sons) 1970.
[4] Jurek, R., Chanussot, J., Bellet, J., C. R. Hebd. Séan. Acad. Sci. B 277 (1973) 53.

[5] Placzek, G., Teller, E., Z. Phys. 81 (1933) 209.

[6] Maes, S., Cah. Phys. 14 (1960) 125. 